\title{
DESIGN AND FABRICATION OF MICRO FLUID POWER SYSTEM BY STEREOLITHOGRAPHY
}

\author{
Yutaka TANAKA*, Naoki TAKEMURA**, Seiya ISHIKURA**, Gaku MINORIKAWA*** \\ * Department of Art \& Technology, Faculty of Engineering \\ **Graduate school of Engineering \\ *** Department of Mechanical Eng., Faculty of Engineering \\ Hosei University \\ 3-7-2 Kajinocho, Koganeishi, Tokyo, 184-8584 Japan \\ (E-mail: y_tanaka@k.hosei.ac.jp)
}

\begin{abstract}
Neither high accumulation nor densification on micro mechatronics are so advanced comparing with semiconductor devices. Our research aims at the establishment of design and fabrication to perform miniaturization, high accumulation and high densification by integrating mechano-micro elements. In difficult assembly of these elements, a stereolithography method is used to form the entire micro fluid power systems in all-in-one design including actuators, pumps and valves. In this paper, prototype models based on a bourdon type and diaphragm type of micro fluid power devices are fabricated and experimentally investigated. Some problems regarding design and fabrication of micro fluid power systems are discussed.
\end{abstract}

\section{KEY WORDS}

Actuator, Bourdon tube, Diaphragm, ECF, Stereolithography

\section{NOMENCLATURE}

$a, b:$ transverse section length diameter, short diameter

$E$ : modulus of elasticity of material

$g$ : acceleration gravity

$h$ : wall thickness of bourdon tube

$P_{a}$ : internal pressure of tube

$P_{p}$ : output pressure of pump

$R$ : curved radius of bourdon tube

$\Delta R$ : change in curved radius

$S:$ displacement of tube tips

$\lambda: a^{2} / R h$

$\theta:$ arc angle of bourdon tube

\section{INTRODUCTION}

In 1989 gear trains and motors on a wafer have been firstly reported by using the semiconductor micro fabrication technology in U.S.A [1]. Semiconductor processing technology applied to these fabrications has touched off since the latter half of 1980's, and the research specialized in microscopic environment has begun to be paid to attention rapidly all over the world. The research on the micro nanotechnology has been carried out as seeds in a wide range of fields such as information and communication technology, mobile machine, manufacturing and inspection, and medicine and biotechnology. Moreover, it is the most important 
technical issue to develop new concept of devices with the best use of characteristics on not only a miniaturization of conventional products but also emphasis of future in microscopic environment.

Recently there are many micro integrated elements such as micro mirrors and acceleration sensors applied to the semiconductor manufacturing process for the practical use [2],[3]. These elements are made on the flat surface of the silicon wafer with fine fabrication and high integration. Therefore, application of these elements has been limited within the practical use of sensors with the control of electronic and optical signals.

On the other hand, neither accumulation nor the integration of various elements in micro mechatronics devices are so advanced comparing with the semiconductor devices. The mechatronics device has functions of sensors, actuators and mechanism, and signal processing. It is difficult for the micro mechatronics device to manufacture and assemble complete parts under microscopic world. Under the microscopic world, adsorption, friction, viscocity and abrasion originated on the surface phenomenon have much influence on the performance, energy efficiency, durability and reliability of the micro mechatronics device.

The component with mechanical moving parts is not suitable for the structure of micro mechatornics system because of large power consumption. Moreover, it is difficult for the micro mechatoronics system to assemble many parts in three dimensional configuration. Therefore, it is preferable to produce it with a complete part where the mechanism is simple and the parts size few as much as possible.

Various actuation principles, for example piezoelectric, electrostatic and thermal expansion have already been reported for the micro actuation system [4]. However, fluid power actuation system has a great potential to realize large output power density comparing to the conventional actuation system under the microscopic environment [5]. In conventional fluid power actuation system, however, there are many mechanical parts in the system, for example valves for actuator control and pumps for power source.

Our research aims at the establishment of design and fabrication to perform miniaturization, high accumulation and high densification by integrating mechano-micro elements. In difficult assembly of these elements, a stereolithography method is used to fabricate a three dimensional structure of the entire micro fluid power systems in all-in-one design.

\section{CONCEPT OF MICRO FLUID POWER SYSTEM}

Our final goal is to develop a new micro fluid power system fabricated by the stereolithography. The device consists of four modules, a signal and sensor module, a

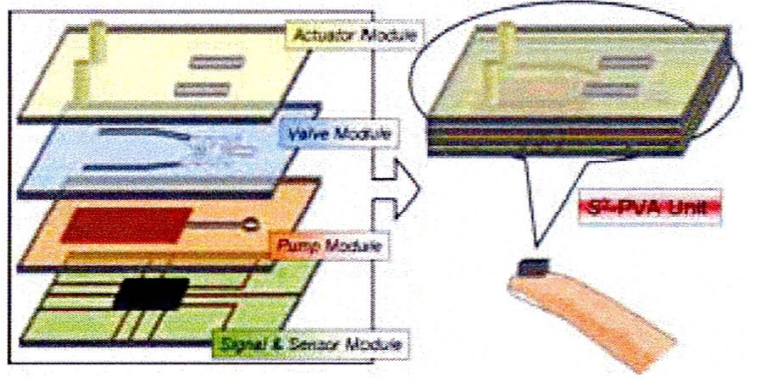

Figure 1 Concept of micro fluid power system

pump module, a valve module, and an actuator module. We call this device the $S^{2}$-PVA unit. Figure 1 shows a concept of the $\mathrm{S}^{2}$-PVA unit with accumulating, integrating, miniaturizing and high performance on one single-chip. This is all-in-one design and fabrication unit consisting of the actuator, the valve, the pump and the sensor modules and functions.

In this paper we report a trial manufactured and an experimental investigated pumping and actuation devices for the actuator module and the pump module by the production technique of the stereolithography.

\section{FABRICATION BY STEREOLITHOGRAPHY}

In the micro processing and micro fabrication, it is difficult to assemble parts on the three dimensional (3D) structure. Prof. Ikuta has pointed out that an optical prototyping has advantage of production for micromachining [6]. Conventional micromachining has made only a two dimensional structure. However, a three-dimensional micro processing becomes possible by using the optical prototyping. Moreover, the optical prototyping method has the potential of processing one time as the internal configuration also included a complex solid model.

In this study, the 3D optical rapid prototyping method, stereolithography, is used to manufacture the each module for the micro fluid power system. Figure 2 shows the optical prototyping process based on a $3 \mathrm{D}$ CAD data. The forming process irradiates the ultraviolet laser $\left(\mathrm{Nd}: \mathrm{YVO}_{4}\right)$ to optical thermosetting property of the liquid based on the $3 \mathrm{D} \mathrm{CAD}$ data, and cures the resin. The 3D shape of prototyping objects can be made by accumulating layers of a certain constant thickness [7]. The stereolithography (3D Systems Co. Viper si2 SLA) used in our research has the accumulating pitch of 50 microns or less. The material property of the resin after curing greatly influences the performance of the fabricated object when the forming thing is used as actuator using the material deformation. The material property after hardening of the resin (the Accura SI 10 Material) used in our experiments are tabulated in Table 1. 


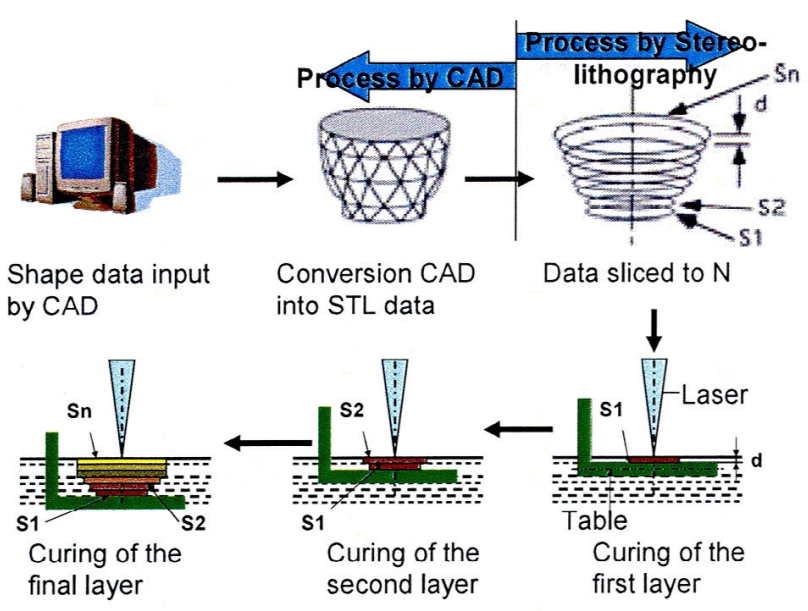

Figure 2 Process of stereolithography

The material property of the resin is measured at final curing after irradiating ultraviolet rays during 90 minutes.

The stereolithography is a new layering technique to fabricate three-dimensional structure of the solid model. A fabricated angle is the important design factor for the stereolithography to fabricate the layered object. Figure 3 shows the fabricated angle of the layered models. Three types of the layered model as shown in Fig.3 are experimentally investigated to consider the fabricated angle of the layer in the stereolithography. The fabricated angle $\psi$ is defined with the respect to the $\mathrm{x}-\mathrm{y}$ plane on the table (horizontal flat surface). The fabricated angles are selected for experiments in the case of $\psi=0^{\circ}, 45^{\circ}$ and $90^{\circ}$, respectively.

Tablel Properties of material

\begin{tabular}{|l|c|}
\hline & Accura SI 10 Material \\
\hline Tensile Strength & $62-63[\mathrm{MPa}]$ \\
\hline Elongation at Break & $3.1-5[\%]$ \\
\hline Tensile Modulus & $3048-3255[\mathrm{MPa}]$ \\
\hline Flexural Strength & $89-97[\mathrm{MPa}]$ \\
\hline Flexural Modulus & $2827-3102[\mathrm{MPa}]$ \\
\hline Impact Strength (Notched Izod) & $18.7-27.7[\mathrm{~J} / \mathrm{m}]$ \\
\hline Glass Transition, Tg & $61.7\left[{ }^{\circ} \mathrm{C}\right]\left(143.1\left[{ }^{\circ} \mathrm{F}\right]\right)$ \\
\hline Hardness, Shore D & 86 \\
\hline
\end{tabular}

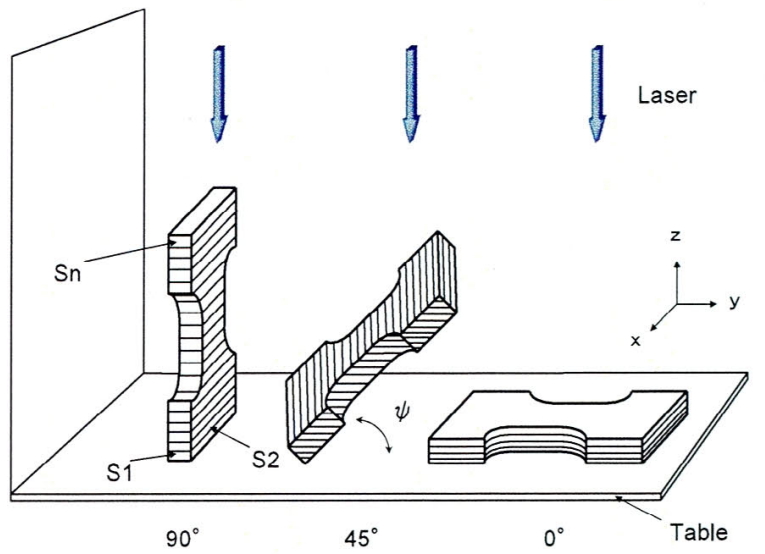

Figure 3 Layer models for fabricated angle

\section{ACTUATOR MODULE}

In this section two types of actuator for the actuator module of the micro fluid power system are described. It has already been shown that the output power density rises as for the fluid actuator under the microscopic environment [5]. Some fluid motors and the elastic deformations using the fluid power have been reported [8], [9]. These actuators have a simple structure and no mechanically operating parts, so that the friction does not influence on the performance of the actuators. Also the power density (power/weight ratio) rises when decreasing dimensions under the microscopic environment. In this paper, we propose bourdon tube type and diaphragm type actuators using the elastic deformation with the fluid power.

\section{Bourdon tube actuator}

Figure 4 shows the geometry of the bourdon tube actuator. Dimensions of the actuator are tabulated in Table 2. The bourdon tube actuator has a high weight torque ratio and small coefficient of friction because of structurally no sliding surface [10], [11]. The bourdon tube actuator is fabricated by the stereolithography to accumulate a number of the layer. The each layer has a thickness of $50 \mu \mathrm{m}$ that is the minimum fabricated layer. A tip of the bourdon tube deforms in response to the applied pressure in the tube. The displacement of the tip $S$ is given by the following Eq.(1).

$$
S=\sqrt{2(1-\cos \theta)-2 \theta \sin \theta+\theta^{2}} \cdot \Delta R
$$

where $\theta$ is the arc angle of the bourdon tube, $\Delta R$ is the change of the bourdon tube curved radius. And the change of the bourdon tube curved radius $\Delta R$ is written as follows. 


$$
\Delta R=\frac{a^{3}}{h^{3}} \frac{K_{2}}{1+\lambda^{2} K_{1}}\left(\frac{P_{a} R}{E}\right)
$$

where $P_{a}$ is the internal pressure in the bourdon tube, $R$ is the radius of the bourdon tube, $E$ is the Young's modulus, $h$ is the wall thickness of the bourdon tube, $a$ is the length of the long axis in the oval across the transverse section, respectively. Coefficients $\lambda\left(=a^{2} / R h\right), K_{1}$ and $K_{2}$ are determined by the shape of the cross-section of the bourdon tube and the length ratio. In this study $K_{1}$ and $K_{2}$ is given as 0.11 and 0.90 , respectively. Moreover, the aspect ratio $v(=b / a)$ is given as 0.43 across the oval section [12].

Table 2 Dimension of bourdon tube

\begin{tabular}{|c|c|c|c|c|c|}
\hline$v(=b / a)$ & $a[\mathrm{~mm}]$ & $b[\mathrm{~mm}]$ & $h[\mathrm{~mm}]$ & $R[\mathrm{~mm}]$ & $\theta[\mathrm{rad}]$ \\
\hline 0.43 & 5.25 & 2.25 & 0.5 & 10 & 4.712 \\
\hline
\end{tabular}
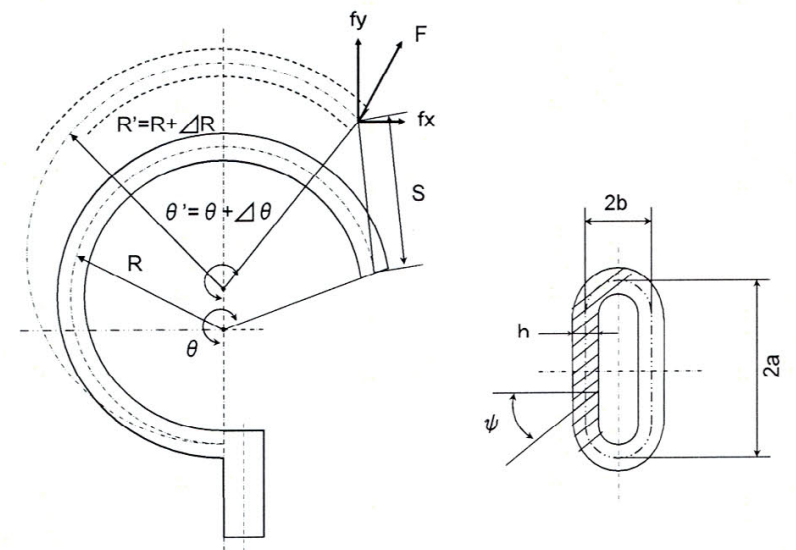

Figure 4 Bourdon tube

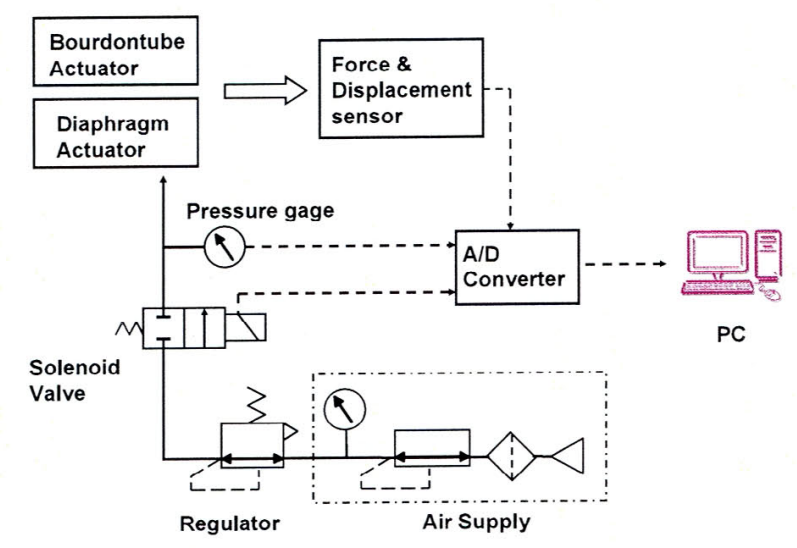

Figure 5 System configurations for experiments

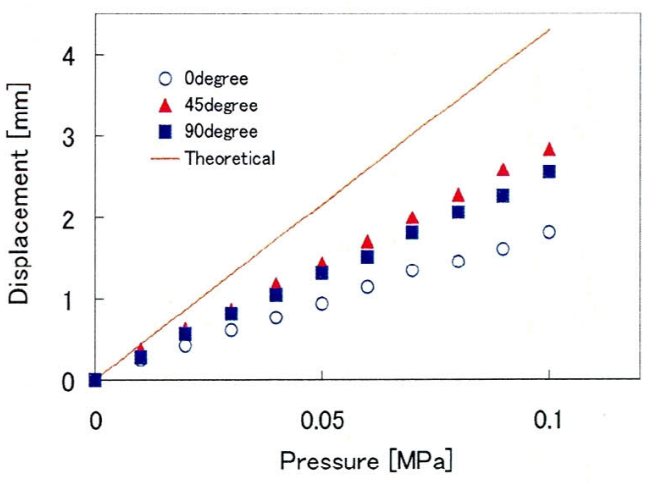

Figure 6 Static characteristics of bourdon tube

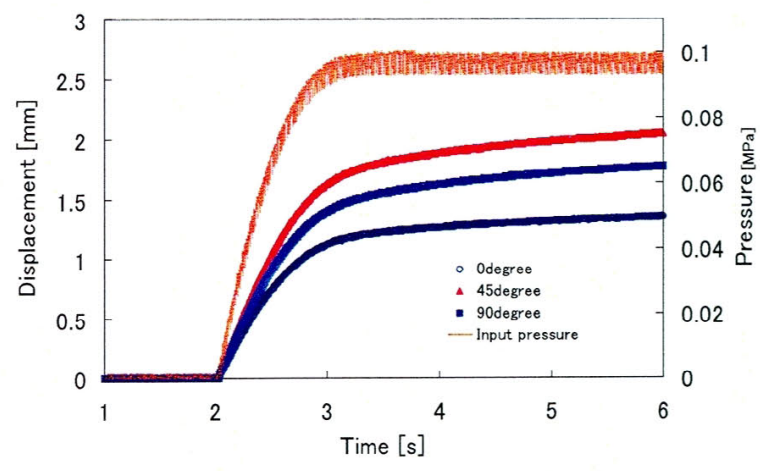

Figure 7 Dynamic characteristic of bourdon tube

Figure 5 shows the system configuration chart used by the fundamental experiment for the characteristics of the actuator. The compressed air is used for the power source in the experiment.

Figure 6 shows the experimental result of the static characteristic of the output displacement of three kinds of bourdon tube actuators with the different fabricated angle. The output displacement is proportional to the supplied pressure. The output displacement nearly depends on the fabricated angle $\psi$. There is no hysterisis property between the supplied pressure and the displacement.

The change of the theoretical displacement calculated from Eq. (1) and Eq. (2) is also plotted in Fig.6. For example the bourdon tube actuator with the fabricated angle $\psi=45^{\circ}$ has the displacement of $2.84 \mathrm{~mm}$ under the supplied pressure condition of $0.1 \mathrm{MPa}$. The experimental value is below $2 / 3$ comparing to the value calculated from Eq. (1). Uniformed materials are assumed to derive the theoretical value by Eq. (1). The structure of the bourdon actuator fabricated by the stereolithography is, however, layered and aeolotropic. Therefore, the fabricated angle influences elastic deformation of the actuator and it causes smaller values compared with the theoretical ones. 
The step signal is applied to the solenoid valve as an evaluation of the dynamic characteristics of the actuator. Figure 7 shows the experimental results for the step response of the actuator. The steady state values in the fabricated angles $\psi=45^{\circ}$ and $90^{\circ}$ are larger than that in the angle $\psi=0^{\circ}$. Thus there are found that the fabricated angle $\psi$ greatly influence on the response of the actuator.

The bourdon tube actuator is easily fabricated by the stereolithography. The output displacement of the actuator and dynamic characteristics are significantly influenced by the direction of the fabricated layer structure. The fabricated angle is one of the important factors to design and fabricate the actuator by the stereolithgraphy.

\section{Diaphragm actuator}

Figure 8 shows geometry of the fabricated diaphragm actuator by stereolithography. The whole film for the diaphragm is made by the curing resin. The shape of the diaphragm is a circle with a diameter of $5 \mathrm{~mm}$ and the film thickness of $0.1 \mathrm{~mm}$. The surface film of diaphragm is fabricated under the condition of only $\psi=90^{\circ}$ for the fabricated layer angle. The film of the diaphragm has a very thin membrane so that the fabrication has resulted in failure in the case of the fabricated angle $\psi=0^{\circ}$ and $45^{\circ}$.

The output displacement and the dynamic characteristic are measured by using the pneumatic system shown in Fig.4 as well as the experiment for the bourdon tube actuator.

Figure 9 shows the experimental results for the static characteristic of the diaphragm actuator. There is a large nonlinear and hysteresis characteristic between the output displacement and supplied pressure to the diaphragm. The internal stress stays in when the resin is cured under the ultraviolet. Figure 10 shows the step response for the diaphragm actuator.

The diaphragm actuator is easily fabricated rather than the bourdon tube actuator. But the performance of the actuator depends on the film of the diaphragm. In this time, the prototype of diaphragm actuator is fabricated as all-in-one design by using the stereolithography. But the stereolithgraphy method using this study could not fabricate thin film.
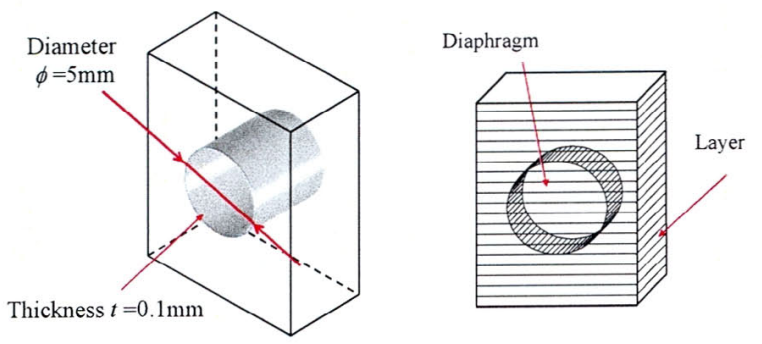

Figure 8 Diaphragm actuator

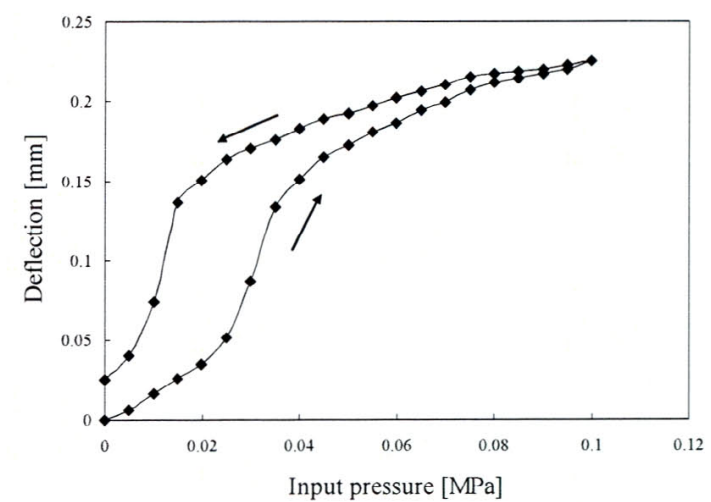

Figure 9 Static characteristics of diaphragm actuator

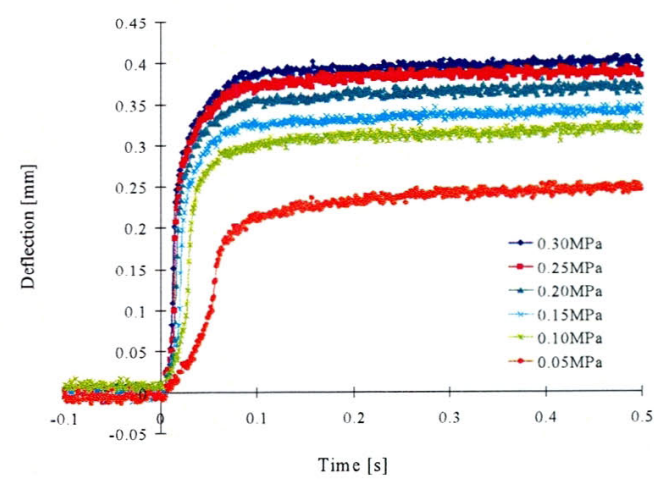

Figure 10 Dynamic characteristics of diaphragm actuator

\section{PUMP MODULE}

In this section we consider about the pump module. Functional fluid ECF (Electro-Conjugate Fluid) is used to be pumping function for the pump module. The ECF occurs the jet flow that is impressed high electrical voltage of direct from a positive electrode to a negative electrode inserted into the fluid [8].

Figure 10 shows structure of the multistage ECF pump module made for trial purposes in this study. A number of electrodes are installed in a pipe with an inner diameter of $2 \mathrm{~mm}$. These electrodes have a spacing of $0.4 \mathrm{~mm}$ and a diameter of $0.1 \mathrm{~mm}$. The coupled electrodes are displayed to the series in $5 \mathrm{~mm}$ spacing for the type of the multistage amplification. Dibutyl decanedioate (DBD) type ECF is used for experiments. The electrical voltage is applied to each couple of electrode from $0 \mathrm{kV}$ to $5 \mathrm{kV}$. The output pressure $\left(P_{p}\right)$ of the actuator is measured by manometer type of pressure sensor.

Figure 12 shows the experimental result of the output pressure using multistage ECF pump. The output pressures have changes of quadratic curve with a little hysteresis according to the applied voltage. Moreover, it 


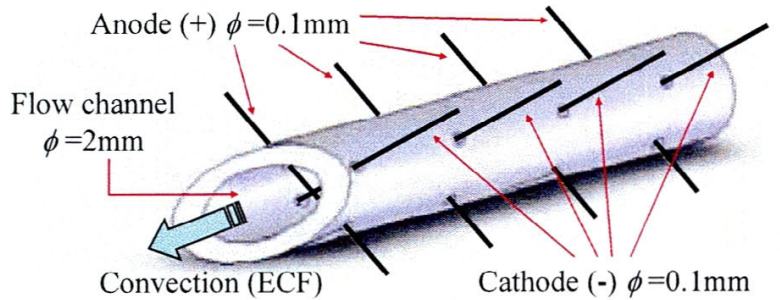

Figure 11 Multistage ECF pump

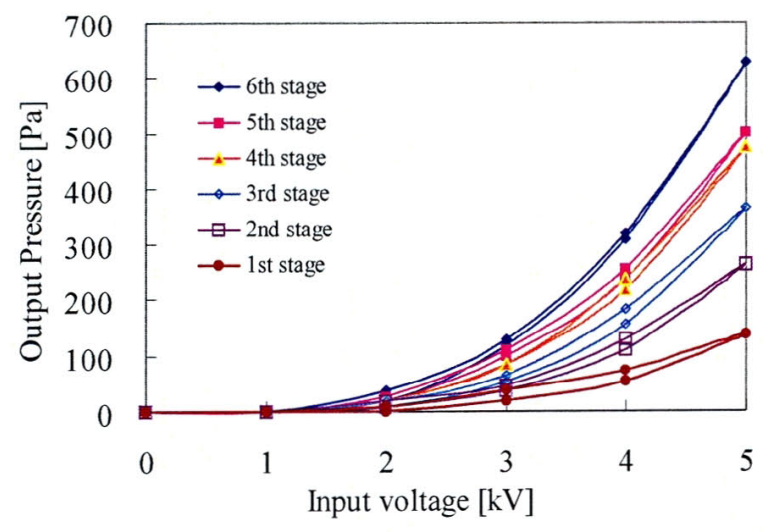

Figure 12 Output pressure for multistage ECF pump

appears to obtain an enough output pressure by increasing number of the electrodes.

It is possible to use the multistage structure of ECF pump as the pump module. The fluid power source using the ECF can be embedded in the main body of the micro systems and can be effective as driving source of the micro actuator. For example these fabricated parts such as actuators and pumps are able to contribute on one single-chip, it may realize to the simplification of the assembly process.

\section{CONCLUSION}

The bourdon tube actuator and the diaphragm actuator are fabricated for trial purposes by using the stereolithography. It is experimentally verified that these actuators have performed by using the fluid power. Moreover the multistage ECF pump is a powerful source to supply necessary power to the actuator for the micro fluid power system. The stereolithography method to fabricate the actuator is the unique technique to integrate and miniaturize some complex parts under the microscopic environment. It is suitable principle for the micro actuator to deform elastically materials without mechanical moving parts. It is clearly pointed out for our experiments that the fabricated angle for the stereolithography is the most important factor to design and fabricate the actuators. It is clarified for all-in-one design unit consisting of the actuators and the pump module to use the stereolithography for fabrication.

\section{Acknowledgement}

This study has been supported in part by Grant-in-Aid for Scientific Research in Priority Areas "next-generation actuators leading break-through" of the ministry of Education, Culture, Sports, Science and Technology of Japan.

\section{REFERENCES}

1. L.S.Fan, Y.C.Tai and R.S.Muller, IC-Processed electrostatic micro motor, Sensor\&Actuators, 1989, 20, pp.41-48.

2. K.D.Wise and K.Najafi, Science 1991, 254, pp.1335-1342.

3. J.M.Bustillo, et al., Proceedinds of the IEEE 86, 1998, pp.1552-1574.

4. Park, J.-H., Yoshida, K. and Yokota, S., Resonantly Driven Piezoelectric Micro Pump-Fabrication of a Micro pump Having High Power density, Mechatronics, Vol.9, No.7, 1999, pp687-702.

5. K.Yoshida and S.Yokota, Study on High-power Micro Actuator Using Fluid Power, Proc. of 6th FLOMEKO, Seoul, 1993, pp.120-130.

6. K.Ikuta, K.Hirowatari, Real Three Dimensional Micro Fabrication Using Stereo Lithography and Metal Molding, Proc. of International Workshop on Micro Electromechanical Systems(MEMS'93), 1993 pp. $42-47$.

7. Jey Won, Kathryn De Laurentis , Constantinos Mavroidis, Rapid Prototyping of Robotic Systems, Proceedinps of the 2000 IEEE, International Conference on Robotics \& Automation, 2000 pp.3077-3082.

8. S.Yokota, Y.Kondoh, A.Sadamoto, Y.Otubo, K.Edamura, A Micro Motor Using Electroconjugate Fluids (Proposition of Stator Electrode-Type (SE-type) Micro ECF motors), JSME International Journal, Vol.44, Ser.C, No.3, 2001, pp.756-762.

9. H. F. Schulte Jr., The Characteristics of the Mckibben Artificial Muscle, The Application of External Power in Prosthetics and Orthetics, pp.94-115.

10. Alfred Wolf, Tuisa Okla, An Elementary Theory of Bourdon Gauge, Journal of Applied Mechanics,Vol.13,No3, 1946, pp209-210.

11. Y.Fujiwara, Y.Saito,K.Hattori : Study of a Pneumatic Robot Utilizing a BTA (Bourdon Tube Actuator), 3rd JHPS International Symposium, 1996, pp.127-132.

12. Flow measurement JSME handbook.4, 1991, pp.7-10, (in Japanese) 